DESY 95-086

ISSN $0418-9833$

hep-ex/yymmnn

May 1995

\title{
A Direct Determination of the Gluon Density in the Proton at Low $\mathrm{x}$
}

\author{
H1 Collaboration
}

\begin{abstract}
A leading order determination of the gluon density in the proton has been performed in the fractional momentum range $1.9 \cdot 10^{-3}<x_{g / p}<0.18$ by measuring multi-jet events from boson-gluon fusion in deep-inelastic scattering with the H1 detector at the electron-proton collider HERA. This direct determination of the gluon density was performed in a kinematic region previously not accessible. The data show a considerable increase of the gluon density with decreasing fractional momenta of the gluons.
\end{abstract}


S. Aid $^{13}$, V. Andreev ${ }^{25}$, B. Andrieu ${ }^{28}$, R.-D. Appuhn ${ }^{11}$, M. Arpagaus ${ }^{36}$, A. Babaev ${ }^{24}$, J. Bähr ${ }^{35}$, J. Bán ${ }^{17}$, Y. Ban ${ }^{27}$, P. Baranov ${ }^{25}$, E. Barrelet ${ }^{29}$, R. Barschke ${ }^{11}$, W. Bartel ${ }^{11}$, M. Barth ${ }^{4}$, U. Bassler ${ }^{29}$, H.P. Beck ${ }^{37}$, H.-J. Behrend ${ }^{11}$, A. Belousov ${ }^{25}$, Ch. Berger ${ }^{1}$, G. Bernardi ${ }^{29}$, R. Bernet ${ }^{36}$, G. Bertrand-Coremans ${ }^{4}$, M. Besançon ${ }^{9}$, R. Beyer ${ }^{11}$, P. Biddulph $^{22}$, P. Bispham ${ }^{22}$, J.C. Bizot ${ }^{27}$, V. Blobel ${ }^{13}$, K. Borras ${ }^{8}$, F. Botterweck ${ }^{4}$, V. Boudry ${ }^{7}$, A. Braemer ${ }^{14}$, F. Brasse ${ }^{11}$, W. Braunschweig ${ }^{1}$, V. Brisson ${ }^{27}$, D. Bruncko ${ }^{17}$, C. Brune ${ }^{15}$, R.Buchholz ${ }^{11}$, L. Büngener ${ }^{13}$, J. Bürger ${ }^{11}$, F.W. Büsser ${ }^{13}$, A. Buniatian ${ }^{11,39}$, S. Burke ${ }^{18}$, M.J. Burton ${ }^{22}$, G. Buschhorn ${ }^{26}$, A.J. Campbell ${ }^{11}$, T. Carli ${ }^{26}$, F. Charles ${ }^{11}$, M. Charlet ${ }^{11}$, D. Clarke $^{5}$, A.B. Clegg ${ }^{18}$, B. Clerbaux ${ }^{4}$, M. Colombo ${ }^{8}$, J.G. Contreras ${ }^{8}$, C. Cormack ${ }^{19}$, J.A. Coughlan ${ }^{5}$, A. Courau ${ }^{27}$, Ch. Coutures ${ }^{9}$, G. Cozzika ${ }^{9}$, L. Criegee ${ }^{11}$, D.G. Cussans ${ }^{5}$, J. Cvach ${ }^{30}$, S. Dagoret ${ }^{29}$, J.B. Dainton ${ }^{19}$, W.D. Dau ${ }^{16}$, K. Daum ${ }^{34}$, M. David $^{9}$, B. Delcourt ${ }^{27}$, L. Del Buono ${ }^{29}$, A. De Roeck ${ }^{11}$, E.A. De Wolf ${ }^{4}$, P. Di Nezza ${ }^{32}$, C. Dollfus ${ }^{37}$, J.D. Dowell ${ }^{3}$, H.B. Dreis ${ }^{2}$, A. Droutskoi ${ }^{24}$, J. Duboc $^{29}$, D. Düllmann ${ }^{13}$, O. Dünger ${ }^{13}$, H. Duhm ${ }^{12}$, J. Ebert $^{34}$, T.R. Ebert ${ }^{19}$, G. Eckerlin ${ }^{11}$, V. Efremenko ${ }^{24}$, S. Egli ${ }^{37}$, H. Ehrlichmann ${ }^{35}$, S. Eichenberger ${ }^{37}$, R. Eichler ${ }^{36}$, F. Eisele ${ }^{14}$, E. Eisenhandler ${ }^{20}$, R.J. Ellison ${ }^{22}$, E. Elsen ${ }^{11}$, M. Erdmann ${ }^{14}$, W. Erdmann ${ }^{36}$, E. Evrard ${ }^{4}$, L. Favart ${ }^{4}$, A. Fedotov ${ }^{24}$, D. Feeken ${ }^{13}$, R. Felst ${ }^{11}$, J. Feltesse ${ }^{9}$, J. Ferencei ${ }^{15}$, F. Ferrarotto ${ }^{32}$, K. Flamm ${ }^{11}$, M. Fleischer ${ }^{26}$, M. Flieser ${ }^{26}$, G. Flügge ${ }^{2}$, A. Fomenko ${ }^{25}$, B. Fominykh ${ }^{24}$, M. Forbush ${ }^{7}$, J. Formánek ${ }^{31}$, J.M. Foster ${ }^{22}$, G. Franke ${ }^{11}$, E. Fretwurst ${ }^{12}$, E. Gabathuler ${ }^{19}$, K. Gabathuler ${ }^{33}$, J. Garvey ${ }^{3}$, J. Gayler ${ }^{11}$, M. Gebauer ${ }^{8}$, A. Gellrich ${ }^{11}$, H. Genzel ${ }^{1}$, R. Gerhards ${ }^{11}$, A. Glazov ${ }^{35}$, U. Goerlach ${ }^{11}$, L. Goerlich ${ }^{6}$, N. Gogitidze ${ }^{25}$, M. Goldberg ${ }^{29}$, D. Goldner ${ }^{8}$, B. Gonzalez-Pineiro ${ }^{29}$, I. Gorelov ${ }^{24}$, P. Goritchev ${ }^{24}$, C. Grab ${ }^{36}$, H. Grässler ${ }^{2}$, R. Grässler ${ }^{2}$, T. Greenshaw ${ }^{19}$, G. Grindhammer ${ }^{26}$, A. Gruber ${ }^{26}$, C. Gruber ${ }^{16}$, J. Haack ${ }^{35}$, D. Haidt ${ }^{11}$, L. Hajduk ${ }^{6}$, O. Hamon ${ }^{29}$, M. Hampel ${ }^{1}$, M. Hapke ${ }^{11}$, W.J. Haynes ${ }^{5}$, J. Heatherington ${ }^{20}$, G. Heinzelmann ${ }^{13}$, R.C.W. Henderson ${ }^{18}$, H. Henschel ${ }^{35}$, I. Herynek ${ }^{30}$, M.F. Hess ${ }^{26}$, W. Hildesheim ${ }^{11}$, P. Hill ${ }^{5}$, K.H. Hiller ${ }^{35}$, C.D. Hilton ${ }^{22}$, J. Hladký $\dot{y}^{30}$, K.C. Hoeger ${ }^{22}$, M. Höppner ${ }^{8}$, R. Horisberger ${ }^{33}$, V.L. Hudgson ${ }^{3}$, Ph. Huet ${ }^{4}$, M. Hütte ${ }^{8}$, H. Hufnagel ${ }^{14}$, M. Ibbotson ${ }^{22}$, H. Itterbeck ${ }^{1}$, M.-A. Jabiol ${ }^{9}$, A. Jacholkowska ${ }^{27}$, C. Jacobsson ${ }^{21}$, M. Jaffre ${ }^{27}$, J. Janoth ${ }^{15}$, T. Jansen ${ }^{11}$, L. Jönsson ${ }^{21}$, D.P. Johnson ${ }^{4}$, L. Johnson ${ }^{18}$, H. Jung ${ }^{29}$, P.I.P. Kalmus ${ }^{20}$, D. Kant ${ }^{20}$, R. Kaschowitz ${ }^{2}$, P. Kasselmann ${ }^{12}$, U. Kathage ${ }^{16}$, J. Katzy ${ }^{14}$, H.H. Kaufmann ${ }^{35}$, S. Kazarian ${ }^{11}$, I.R. Kenyon ${ }^{3}$, S. Kermiche ${ }^{23}$, C. Keuker ${ }^{1}$, C. Kiesling ${ }^{26}$, M. Klein ${ }^{35}$, C. Kleinwort ${ }^{13}$, G. Knies ${ }^{11}$, W. Ko ${ }^{7}$, T. Köhler ${ }^{1}$, J.H. Köhne ${ }^{26}$, H. Kolanoski ${ }^{8}$, F. Kole ${ }^{7}$, S.D. Kolya ${ }^{22}$, V. Korbel ${ }^{11}$, M. Korn ${ }^{8}$, P. Kostka ${ }^{35}$, S.K. Kotelnikov ${ }^{25}$, T. Krämerkämper ${ }^{8}$, M.W. Krasny ${ }^{6,29}$, H. Krehbiel ${ }^{11}$, D. Krücker ${ }^{2}$, U. Krüger ${ }^{11}$, U. Krüner-Marquis ${ }^{11}$, H. Küster ${ }^{2}$, M. Kuhlen ${ }^{26}$, T. Kurča ${ }^{17}$, J. Kurzhöfer ${ }^{8}$, B. Kuznik ${ }^{34}$, D. Lacour ${ }^{29}$, F. Lamarche ${ }^{28}$, R. Lander ${ }^{7}$, M.P.J. Landon ${ }^{20}$, W. Lange ${ }^{35}$, P. Lanius ${ }^{26}$, J.-F. Laporte ${ }^{9}$, A. Lebedev ${ }^{25}$, F. Lehner ${ }^{11}$, C. Leverenz ${ }^{11}$, S. Levonian ${ }^{25}$, Ch. Ley ${ }^{2}$, A. Lindner ${ }^{8}$, G. Lindström ${ }^{12}$, J. Link ${ }^{7}$, F. Linsel ${ }^{11}$, J. Lipinski ${ }^{13}$,

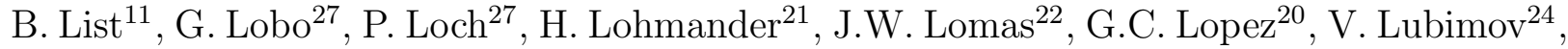
D. Lüke ${ }^{8,11}$, N. Magnussen ${ }^{34}$, E. Malinovski ${ }^{25}$, S. Mani ${ }^{7}$, R. Maraček ${ }^{17}$, P. Marage ${ }^{4}$, J. Marks ${ }^{23}$, R. Marshall 22 , J. Martens ${ }^{34}$, G. Martin ${ }^{13}$, R. Martin ${ }^{11}$, H.-U. Martyn ${ }^{1}$, J. Martyniak ${ }^{27}$, S. Masson ${ }^{2}$, T. Mavroidis ${ }^{20}$, S.J. Maxfield ${ }^{19}$, S.J. McMahon ${ }^{19}$, A. Mehta ${ }^{22}$, K. Meier ${ }^{15}$, D. Mercer ${ }^{22}$, T. Merz ${ }^{35}$, A. Meyer ${ }^{11}$, C.A. Meyer ${ }^{37}$, H. Meyer ${ }^{34}$, J. Meyer ${ }^{11}$, A. Migliori28, S. Mikocki ${ }^{6}$, D. Milstead ${ }^{19}$, F. Moreau ${ }^{28}$, J.V. Morris ${ }^{5}$, E. Mroczko ${ }^{6}$, G. Müller ${ }^{11}$, K. Müller ${ }^{11}$, P. Murín ${ }^{17}$, V. Nagovizin ${ }^{24}$, R. Nahnhauer ${ }^{35}$, B. Naroska ${ }^{13}$, Th. Naumann ${ }^{35}$, P.R. Newman ${ }^{3}$, D. Newton ${ }^{18}$, D. Neyret ${ }^{29}$, H.K. Nguyen ${ }^{29}$, T.C. Nicholls ${ }^{3}$, F. Niebergall ${ }^{13}$, C. Niebuhr ${ }^{11}$, Ch. Niedzballa ${ }^{1}$, R. Nisius ${ }^{1}$, G. Nowak ${ }^{6}$, G.W. Noyes ${ }^{5}$, M. Nyberg-Werther ${ }^{21}$, M. Oakden ${ }^{19}$, 
H. Oberlack ${ }^{26}$, U. Obrock ${ }^{8}$, J.E. Olsson ${ }^{11}$, D. Ozerov ${ }^{24}$, E. Panaro ${ }^{11}$, A. Panitch ${ }^{4}$, C. Pascaud ${ }^{27}$, G.D. Patel ${ }^{19}$, E. Peppel ${ }^{35}$, E. Perez ${ }^{9}$, J.P. Phillips ${ }^{22}$, Ch. Pichler ${ }^{12}$, A. Pieuchot ${ }^{23}$, D. Pitzl ${ }^{36}$, G. Pope ${ }^{7}$, S. Prell ${ }^{11}$, R. Prosi ${ }^{11}$, K. Rabbertz ${ }^{1}$, G. Rädel ${ }^{11}$, F. Raupach ${ }^{1}$, P. Reimer ${ }^{30}$, S. Reinshagen ${ }^{11}$, P. Ribarics ${ }^{26}$, H.Rick ${ }^{8}$, V. Riech ${ }^{12}$, J. Riedlberger ${ }^{36}$, S. Riess ${ }^{13}$, M. Rietz ${ }^{2}$, E. Rizvi ${ }^{20}$, S.M. Robertson ${ }^{3}$, P. Robmann ${ }^{37}$, H.E. Roloff ${ }^{35}$, R. Roosen ${ }^{4}$, K. Rosenbauer ${ }^{1}$ A. Rostovtsev ${ }^{24}$, F. Rouse ${ }^{7}$, C. Royon ${ }^{9}$, K. Rüter ${ }^{26}$, S. Rusakov ${ }^{25}$, K. Rybicki $^{6}$, R. Rylko $^{20}$, N. Sahlmann ${ }^{2}$, D.P.C. Sankey ${ }^{5}$, P. Schacht ${ }^{26}$, S. Schiek ${ }^{13}$, S. Schleif ${ }^{15}$, P. Schleper ${ }^{14}$, W. von Schlippe ${ }^{20}$, D. Schmidt ${ }^{34}$, G. Schmidt ${ }^{13}$, A. Schöning ${ }^{11}$, V. Schröder ${ }^{11}$, E. Schuhmann ${ }^{26}$, B. Schwab ${ }^{14}$, G. Sciacca ${ }^{35}$, F. Sefkow ${ }^{11}$, M. Seidel ${ }^{12}$, R. Sell ${ }^{11}$, A. Semenov ${ }^{24}$, V. Shekelyan ${ }^{11}$, I. Sheviakov ${ }^{25}$, L.N. Shtarkov ${ }^{25}$, G. Siegmon ${ }^{16}$, U. Siewert ${ }^{16}$, Y. Sirois ${ }^{28}$, I.O. Skillicorn ${ }^{10}$, P. Smirnov ${ }^{25}$, J.R. Smith ${ }^{7}$, V. Solochenko ${ }^{24}$, Y. Soloviev ${ }^{25}$, J. Spiekermann ${ }^{8}$, S. Spielman ${ }^{28}$, H. Spitzer ${ }^{13}$, R. Starosta ${ }^{1}$, M. Steenbock ${ }^{13}$, P. Steffen ${ }^{11}$, R. Steinberg ${ }^{2}$, B. Stella ${ }^{32}$, K. Stephens ${ }^{22}$, J. Stier ${ }^{11}$, J. Stiewe ${ }^{15}$, U. Stößlein ${ }^{35}$, K. Stolze ${ }^{35}$, J. Strachota ${ }^{30}$, U. Straumann ${ }^{37}$, W. Struczinski ${ }^{2}$, J.P. Sutton ${ }^{3}$, S. Tapprogge ${ }^{15}$, V. Tchernyshov ${ }^{24}$, C. Thiebaux $^{28}$, G. Thompson ${ }^{20}$, P. Truöl ${ }^{37}$, J. Turnau ${ }^{6}$, J. Tutas ${ }^{14}$, P. Uelkes ${ }^{2}$, A. Usik ${ }^{25}$, S. Valkár ${ }^{31}$, A. Valkárová31, C. Vallée ${ }^{23}$, D. Vandenplas ${ }^{28}$, P. Van $\mathrm{Esch}^{4}$, P. Van Mechelen ${ }^{4}$, A. Vartapetian ${ }^{11,39}$, Y. Vazdik ${ }^{25}$, P. Verrecchia ${ }^{9}$, G. Villet ${ }^{9}$, K. Wacker $^{8}$, A. Wagener ${ }^{2}$, M. Wagener ${ }^{33}$, A. Walther ${ }^{8}$, G. Weber ${ }^{13}$, M. Weber ${ }^{11}$, D. Wegener ${ }^{8}$, A. Wegner ${ }^{11}$, H.P. Wellisch ${ }^{26}$, L.R. West ${ }^{3}$, S. Willard ${ }^{7}$, M. Winde ${ }^{35}$, G.-G. Winter ${ }^{11}$, C. Wittek ${ }^{13}$, A.E. Wright ${ }^{22}$, E. Wünsch ${ }^{11}$, N. Wulffl ${ }^{11}$, T.P. Yiou ${ }^{29}$, J. Žáček ${ }^{31}$, D. Zarbock ${ }^{12}$, Z. Zhang ${ }^{27}$, A. Zhokin²4, M. Zimmer ${ }^{11}$, W. Zimmermann ${ }^{11}$, F. Zomer ${ }^{27}$, K. Zuber ${ }^{15}$, and M. zur Nedden ${ }^{37}$

${ }^{1}$ I. Physikalisches Institut der RWTH, Aachen, Germany ${ }^{a}$

${ }^{2}$ III. Physikalisches Institut der RWTH, Aachen, Germany ${ }^{a}$

${ }^{3}$ School of Physics and Space Research, University of Birmingham, Birmingham, UK ${ }^{b}$

${ }^{4}$ Inter-University Institute for High Energies ULB-VUB, Brussels; Universitaire Instelling Antwerpen, Wilrijk, Belgium ${ }^{c}$

${ }^{5}$ Rutherford Appleton Laboratory, Chilton, Didcot, $\mathrm{UK}^{b}$

${ }^{6}$ Institute for Nuclear Physics, Cracow, Poland ${ }^{d}$

${ }^{7}$ Physics Department and IIRPA, University of California, Davis, California, USA ${ }^{e}$

${ }^{8}$ Institut für Physik, Universität Dortmund, Dortmund, Germany ${ }^{a}$

9 CEA, DSM/DAPNIA, CE-Saclay, Gif-sur-Yvette, France

10 Department of Physics and Astronomy, University of Glasgow, Glasgow, UK ${ }^{b}$

${ }^{11}$ DESY, Hamburg, Germany ${ }^{a}$

12 I. Institut für Experimentalphysik, Universität Hamburg, Hamburg, Germany ${ }^{a}$

${ }^{13}$ II. Institut für Experimentalphysik, Universität Hamburg, Hamburg, Germany ${ }^{a}$

${ }^{14}$ Physikalisches Institut, Universität Heidelberg, Heidelberg, Germany ${ }^{a}$

${ }^{15}$ Institut für Hochenergiephysik, Universität Heidelberg, Heidelberg, Germany ${ }^{a}$

16 Institut für Reine und Angewandte Kernphysik, Universität Kiel, Kiel, Germany ${ }^{a}$

${ }^{17}$ Institute of Experimental Physics, Slovak Academy of Sciences, Košice, Slovak Republic ${ }^{f}$

18 School of Physics and Chemistry, University of Lancaster, Lancaster, $\mathrm{UK}^{b}$

19 Department of Physics, University of Liverpool, Liverpool, $\mathrm{UK}^{b}$ 
20 Queen Mary and Westfield College, London, $\mathrm{UK}^{b}$

21 Physics Department, University of Lund, Lund, Sweden ${ }^{g}$

22 Physics Department, University of Manchester, Manchester, $\mathrm{UK}^{b}$

${ }^{23}$ CPPM, Université d'Aix-Marseille II, IN2P3-CNRS, Marseille, France

${ }^{24}$ Institute for Theoretical and Experimental Physics, Moscow, Russia

${ }^{25}$ Lebedev Physical Institute, Moscow, Russia ${ }^{f}$

${ }^{26}$ Max-Planck-Institut für Physik, München, Germany ${ }^{a}$

27 LAL, Université de Paris-Sud, IN2P3-CNRS, Orsay, France

${ }^{28}$ LPNHE, Ecole Polytechnique, IN2P3-CNRS, Palaiseau, France

${ }^{29}$ LPNHE, Universités Paris VI and VII, IN2P3-CNRS, Paris, France

${ }^{30}$ Institute of Physics, Czech Academy of Sciences, Praha, Czech Republic ${ }^{f, h}$

31 Nuclear Center, Charles University, Praha, Czech Republic ${ }^{f, h}$

32 INFN Roma and Dipartimento di Fisica, Universita "La Sapienza", Roma, Italy

${ }^{33}$ Paul Scherrer Institut, Villigen, Switzerland

34 Fachbereich Physik, Bergische Universität Gesamthochschule Wuppertal, Wuppertal, Germany $^{a}$

${ }^{35}$ DESY, Institut für Hochenergiephysik, Zeuthen, Germany ${ }^{a}$

${ }^{36}$ Institut für Teilchenphysik, ETH, Zürich, Switzerland ${ }^{i}$

37 Physik-Institut der Universität Zürich, Zürich, Switzerland ${ }^{i}$

38 Stanford Linear Accelerator Center, Stanford California, USA

${ }^{39}$ Visitor from Yerevan Phys.Inst., Armenia

a Supported by the Bundesministerium für Bildung und Forschung, FRG under contract numbers 6AC17P, 6AC47P, 6DO57I, 6HH17P, 6HH27I, 6HD17I, 6HD27I, 6KI17P, 6MP17I, and 6WT87P

${ }^{b}$ Supported by the UK Particle Physics and Astronomy Research Council, and formerly by the UK Science and Engineering Research Council

${ }^{c}$ Supported by FNRS-NFWO, IISN-IIKW

${ }^{d}$ Supported by the Polish State Committee for Scientific Research, grant No. 204209101

e Supported in part by USDOE grant DE F603 91ER40674

${ }^{f}$ Supported by the Deutsche Forschungsgemeinschaft

${ }^{g}$ Supported by the Swedish Natural Science Research Council

${ }^{h}$ Supported by GA ČR, grant no. 202/93/2423, GA AV ČR, grant no. 19095 and GA UK, grant no. 342

${ }^{i}$ Supported by the Swiss National Science Foundation 


\section{Introduction}

Deep-inelastic lepton-nucleon scattering experiments have played a fundamental role in reaching an understanding of the structure of matter. Ever since the discovery of the proton's parton content in the late 1960s, extensive studies have been made at accelerators providing increasingly higher energies to obtain more detailed knowledge of the parton properties within nucleons. Although it was soon realized that about $50 \%$ of the nucleon momentum was carried by gluons, a direct measurement of their momentum distribution has so far been restricted to large fractional momenta. Instead information about the gluons has been extracted from measurements of the sea-quark distribution. This relies on the assumption that sea-quark pairs are produced by quark-antiquark production from gluons in an evolution process.
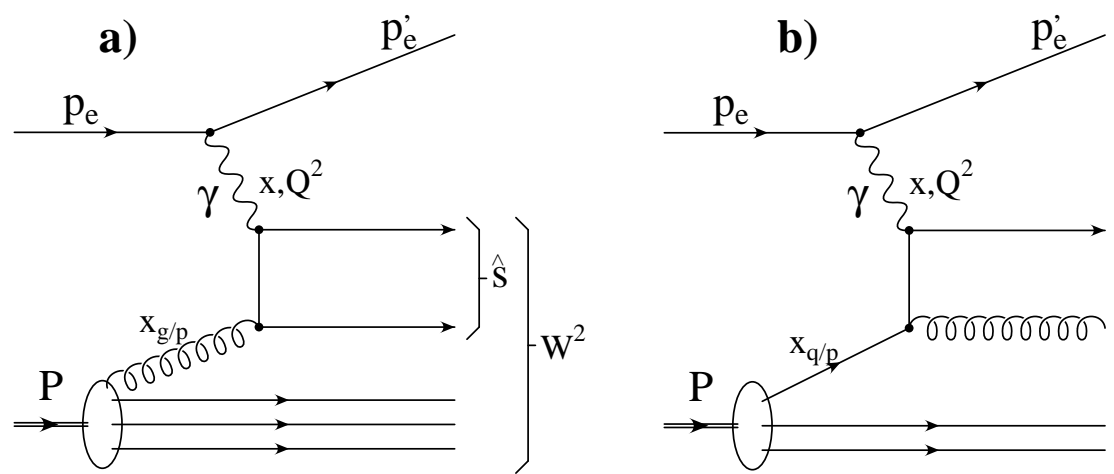

Figure 1: Generic Feynman diagrams for a) the boson-gluon fusion process and, b) the QCD-Compton process

The electron-proton collider HERA has considerably extended the kinematic region available to investigations of nucleon constituents. In particular it permits measurements of processes directly initiated by gluons in the proton. In the boson-gluon fusion process (Fig. 1a) a gluon from the proton interacts with the virtual boson from the electron to produce a quark-antiquark pair. In the range of momentum transfer $\left(Q^{2}\right)$ considered in this analysis photon exchange is dominant and the contribution from $Z^{\circ}$ exchange can be neglected. Typically the final state contains two jets in addition to the proton fragment which, to a large extent, disappears undetected down the beam pipe. Such events are denoted as $(2+1)$ jet events. The hard sub-system is the system produced by the interaction of the virtual photon with a parton in the proton. The $(2+1)$ jet cross section also receives a contribution from the QCD-Compton process (Fig. 1b) where a gluon is emitted from the interacting quark. The cross section of $(2+1)$ jet events can in leading order schematically be written as

$$
\sigma_{2+1} \propto \alpha_{s}(A \cdot g+B \cdot q)
$$

where $\alpha_{s}$ is the strong coupling constant and $g$ and $q$ stand for the gluon and quark densities. The coefficients $A$ and $B$ can be calculated in pertubative QCD. The $(2+1)$ 
jet rate at HERA offers a possibility to determine the variation of the strong coupling constant with the momentum transfer of the exchanged boson. Such a study has been carried out [1] based on the assumption that the quark and gluon densities in the proton are sufficiently constrained at lower momentum transfer by previous experiments. The analysis of the gluon density presented here, on the other hand, relies upon a reasonably good knowledge about the strong coupling constant and the quark densities. In the kinematic region used for this analysis, the multi-jet processes are initiated by partons carrying a momentum fraction of the proton of the order of $10^{-2}$, a region in which the quark densities have been accurately measured in previous experiments [2, [3].

\section{The H1 Detector}

A detailed description of the $\mathrm{H} 1$ detector can be found elsewhere [4]. Closest to the interaction point there are tracking devices surrounded by a calorimeter consisting of an electromagnetic and a hadronic section. Outside these detectors a superconducting coil provides a magnetic field parallel to the beam line and, finally, the instrumented magnet iron gives a rough measurement of the energy leaking out of the calorimeter, and signals the presence of a muon track. Here we only give a brief description of the detector parts which are of relevance to the measurement of the gluon density.

The backward electromagnetic calorimeter (BEMC) covers the angular range $155^{\circ}<$ $\theta<176^{\circ}$ where $\theta$ is defined with respect to the proton beam direction, which is in the following called the forward direction. Making use of the track information from the backward proportional wire chamber (BPC), located right in front of the BEMC, and the reconstructed vertex position, the energy and scattering angle of the scattered electron can be measured in the kinematic region considered here. The BEMC consists of a 22.5 radiation length deep lead-scintillator sandwich stack, each layer read out by two pairs of oppositely positioned wavelength shifter bars. This system gives an energy resolution of $\sigma(E) / E \approx 0.1 / \sqrt{E[\mathrm{GeV}]} \oplus 0.42 / E[\mathrm{GeV}] \oplus 0.03$. By adjusting the measured electron energy spectrum to the kinematic peak, the BEMC energy calibration is known to an accuracy of $1.7 \%$ [5]. The BPC has four wire planes, giving a spatial resolution of 1.5 $\mathrm{mm}$, which together with the precision in the vertex reconstruction, results in a resolution of the polar angle of $2.5 \mathrm{mrad}$.

For the measurement of the hadronic final state the liquid argon calorimeter was used, which extends over the angular range $4^{\circ}<\theta<153^{\circ}$ with a complete azimuthal coverage. The total depth varies between 4.5 and 8 interaction lengths. Measurements

on test beams have given a hadronic energy resolution of $\sigma(E) / E \approx 0.5 / \sqrt{E[\mathrm{GeV}]} \oplus 0.02$ [6]. The absolute hadronic energy scale has been measured to a precision of $5 \%$ from the transverse momentum balance between hadronic jets and the scattered electron in deep-inelastic scattering (DIS) events. 


\section{Kinematics}

In 1993 HERA was operated to collide $26.7 \mathrm{GeV}$ electrons with $820 \mathrm{GeV}$ protons, resulting in a center-of-mass energy of $296 \mathrm{GeV}$. Fig. 1 shows Feynman diagrams of the bosongluon fusion process (BGF) and the QCD-Compton process (QCD-C) with the relevant kinematic variables indicated.

The overall kinematics of an event can be determined from two independent Lorentz invariant variables. This can be any two of the Bjørken scaling variables $x$ and $y$, the photon momentum transfer squared $Q^{2}$, and the invariant mass squared of the hadronic system $W^{2}$. These variables are defined in terms of the four-momenta of the incoming proton, $P$, the incoming and outgoing electron, $p_{e}$ and $p_{e}^{\prime}$, and the exchanged photon, $q$. Experimentally they are deduced from measurements of the energy, $E_{e}^{\prime}$, and polar angle, $\theta_{e}$, of the scattered electron according to the following relations (neglecting the electron and proton masses):

$$
\begin{aligned}
& Q^{2} \equiv-q^{2}=-\left(p_{e}-p_{e}^{\prime}\right)^{2}=4 E_{e} E_{e}^{\prime} \cos ^{2}\left(\theta_{e} / 2\right) \\
& y \equiv \frac{P \cdot q}{P \cdot p_{e}}=1-\left(E_{e}^{\prime} / E_{e}\right) \sin ^{2}\left(\theta_{e} / 2\right) \\
& x \equiv \frac{-q^{2}}{2 P \cdot q}=\frac{Q^{2}}{y s} ; \quad W^{2}=Q^{2}\left(\frac{1-x}{x}\right)
\end{aligned}
$$

The center-of-mass energy squared is given by $s=4 E_{e} E_{p}$, where $E_{e}$ and $E_{p}$ are the energies of the incoming electron and proton, respectively. Note that Bjørken- $x$ is not identical to the fractional momentum $x_{i / p}$ of the parton $i$ initiating the hard sub-process, with $i$ standing for either a quark $(q)$ or a gluon $(g) . x_{i / p}$ is related to the invariant mass squared of the hard sub-system $(\hat{s})$ in the following way:

$$
x_{i / p}=\frac{\hat{s}+Q^{2}}{y s}=x\left(1+\frac{\hat{s}}{Q^{2}}\right)
$$

where $\hat{s}$ can be measured either directly from the invariant mass of all particles $p_{j}$ of the two jets belonging to the hard sub-system

$$
\hat{s}=\left(\sum_{j} p_{j}\right)^{2}
$$

or from the jet directions in the hadronic center-of-mass system, which is the rest system of the exchanged photon and the proton:

$$
\hat{s}=W^{2} e^{-\left(\eta_{1}^{*}+\eta_{2}^{*}\right)}
$$

where $\eta_{1}^{*}$ and $\eta_{2}^{*}$ are the parton pseudo-rapidities of the hard sub-process $\emptyset$ and $\eta=$ $-\ln \tan (\theta / 2)$. Experimentally the parton directions and momenta are approximated by the directions and energies of the jets produced in the fragmentation process.

${ }^{1}$ Variables $v$ in the hadronic center of mass system are denoted as $v^{*}$ while variables in the photonparton center of mass frame are denoted as $\hat{v}$. The direction of the $z$-axis in the hadronic center of mass system is defined as the direction of the exchanged photon. Note that the transverse momentum $p_{T}^{*}=\hat{p}_{T}$. 


\section{Trigger and Data Selection}

The analysis is performed with deep-inelastic scattering (DIS) events which are selected in the detector by applying the trigger requirement for an electron of a local energy deposition (cluster) of more than $4 \mathrm{GeV}$ in the BEMC detector. The total event sample used in this analysis corresponds to an integrated luminosity of $242 \mathrm{nb}^{-1}$ from the data sample collected in 1993. After the event reconstruction has been performed, a clean DIS sample is obtained by applying the following requirements:

- The scattered electron energy had to satisfy $E_{e}^{\prime}>10 \mathrm{GeV}$ which corresponds to $y \lesssim 0.625$. This removes most of the background due to the photoproduction reactions where an electromagnetic shower in the hadronic final state is misidentified as an electron from the DIS process.

- The track coordinates in the BPC had to match the cluster center-of-gravity in the BEMC to within $4 \mathrm{~cm}$ and the lateral spread of the cluster had to be less than $4 \mathrm{~cm}$ in radius.

- The momentum transfer squared had to be in the range $12.5<Q^{2}<80 \mathrm{GeV}^{2}$ which ensures that the scattered electron is well contained in the BEMC.

- In order to get an adequate resolution in $y$ from the measurement of the scattered electron it was required that $y>0.05$.

- At least one charged track from the hadronic final state was demanded for the determination of the vertex position along the beam, which had to be within $30 \mathrm{~cm}$ from the nominal position in order to suppress beam-induced background.

After these cuts and the jet selection described below no indication was observed of remaining background from photoproduction in our data. This was confirmed by a Monte Carlo simulation of photoproduction processes.

Efficiencies for passing the above cuts have been determined from data using the redundancy in our apparatus. Further details can be found in [5]. The $4 \%$ error in the combined efficiency will contribute to the overall normalization error.

The $(2+1)$ jet selection is carried out in two steps. In the first step the jet finding is performed by applying a cone algorithm [7] on calorimeter clusters with $\Delta R=$ $\sqrt{\Delta \eta^{2}+\Delta \Phi^{2}}=1$ first boosting to the hadronic center-of-mass system. $\Delta \eta$ and $\Delta \Phi$ are the differences in pseudo-rapidity and azimuthal angle between two cluster pairs respectively. Exactly two jets with a transverse jet energy $p_{T}^{*}>3.5 \mathrm{GeV}$ are required. The hadronic center-of-mass system is a natural frame for a QCD analysis as it is not sensitive to the $p_{T}$ of the hadronic system due to the transverse momentum balance of the electron in the laboratory system. It is also a preferred frame for the $p_{T}$ based cone algorithm. The ratio of $(3+1)$ to $(2+1)$ jet events is less than $15 \%$.

In the second step following cuts are imposed on the jet system of the event:

- Both reconstructed jets have to fall inside the angular range $10^{\circ}<\theta_{\text {jet }}<150^{\circ}$ in the laboratory system so as to be within the volume covered by the liquid argon calorimeter. 
A further purpose of the lower cut in angle is to remove the very forward region which is dominated by the proton fragments and initial state parton radiation which may otherwise give rise to a separate jet.

- The difference in pseudo-rapidity between the two jets in the laboratory frame must satisfy $\Delta \eta<2$. This corresponds to a cut on the scattering angle in the photon-parton center of mass system of $|\hat{\eta}| \lesssim 1$ or $40^{\circ} \lesssim \hat{\theta} \lesssim 140^{\circ}$ in the kinematic range used for this analysis. Note that this cut, except for the highest $x_{g / p}$ covered by this analysis, is more restrictive than the angular cut discussed above.

- The corrected invariant mass (see section 6) of the two jets of the hard scattering system is required to be $\sqrt{\hat{s}}>10 \mathrm{GeV}$ to ensure well defined jet structures (see Fig. 2). This cut, together with the jet angular cut $\Delta \eta<2$, implies $p_{T}^{*} \gtrsim 3.2 \mathrm{GeV}$ and is therefore well matched to the $p_{T}^{*}$ cut of $3.5 \mathrm{GeV}$ in the application of the cone algorithm. In addition both methods are used to reconstruct $\hat{s}$, as given by equations (2) and (3). It is required that the difference in $|\Delta \sqrt{\hat{s}}|$ for the two methods is smaller than $10 \mathrm{GeV}$.

With these cuts a sample of $328(2+1)$ jet events covering the fractional momentum range $0.002 \lesssim x_{g / p} \lesssim 0.2$ and the Bjørken- $x$ range $0.0003 \lesssim x \lesssim .0015$ is obtained. In the kinematic region which was chosen for this analysis, according to the cuts defined above, the ratio of $\mathrm{BGF}$ and $\mathrm{QCD}-\mathrm{Compton}$ cross sections is approximately 3/1, independent of $x_{i / p}$.

\section{Monte Carlo Generation}

In order to calculate cross sections from matrix elements and to correct for parton shower, hadronisation, and detector effects, the Monte Carlo event generator LEPTO 6.1 [8] was used, with the parton density function MRS H [9]. Although it would have been more natural for this analysis to use leading order parametrisations of the density functions it will be explained in the discussion of errors (section 7) that this is not essential. The strong coupling constant $\alpha_{s}$ was calculated in first order QCD with $\Lambda_{Q C D}=250 \mathrm{MeV}$ for 5 flavours. It was checked that LEPTO delivers cross sections which are consistent with leading order cross section predictions by the PROJET [10] and DISJET [11] programs within $5 \%$ in the kinematic region covered by this analysis.

The LEPTO generator is based on QCD matrix element calculations up to first order in $\alpha_{s}$, and the inclusion of parton showers accounts for higher order processes (MEPS). The QCD matrix element gives divergences for soft and collinear emission which is technically avoided by defining a smallest invariant mass $m_{i j}$ between any two partons, including the remnant. The parametrisation used for the cut in $m_{i j}$ is always $2 \mathrm{GeV}$ above the region in phase space where the $2+1$ jet cross section would exceed the total cross section. With such a cut, matrix element calculations are used to generate events over the maximum

possible phase space, thereby giving access to regions of small $x_{g / p}$. The Monte Carlo matrix element cut-off is always significantly below $10 \mathrm{GeV}$ which is the constant mass cut applied in the event selection. Therefore the full phase space used in this analysis is 

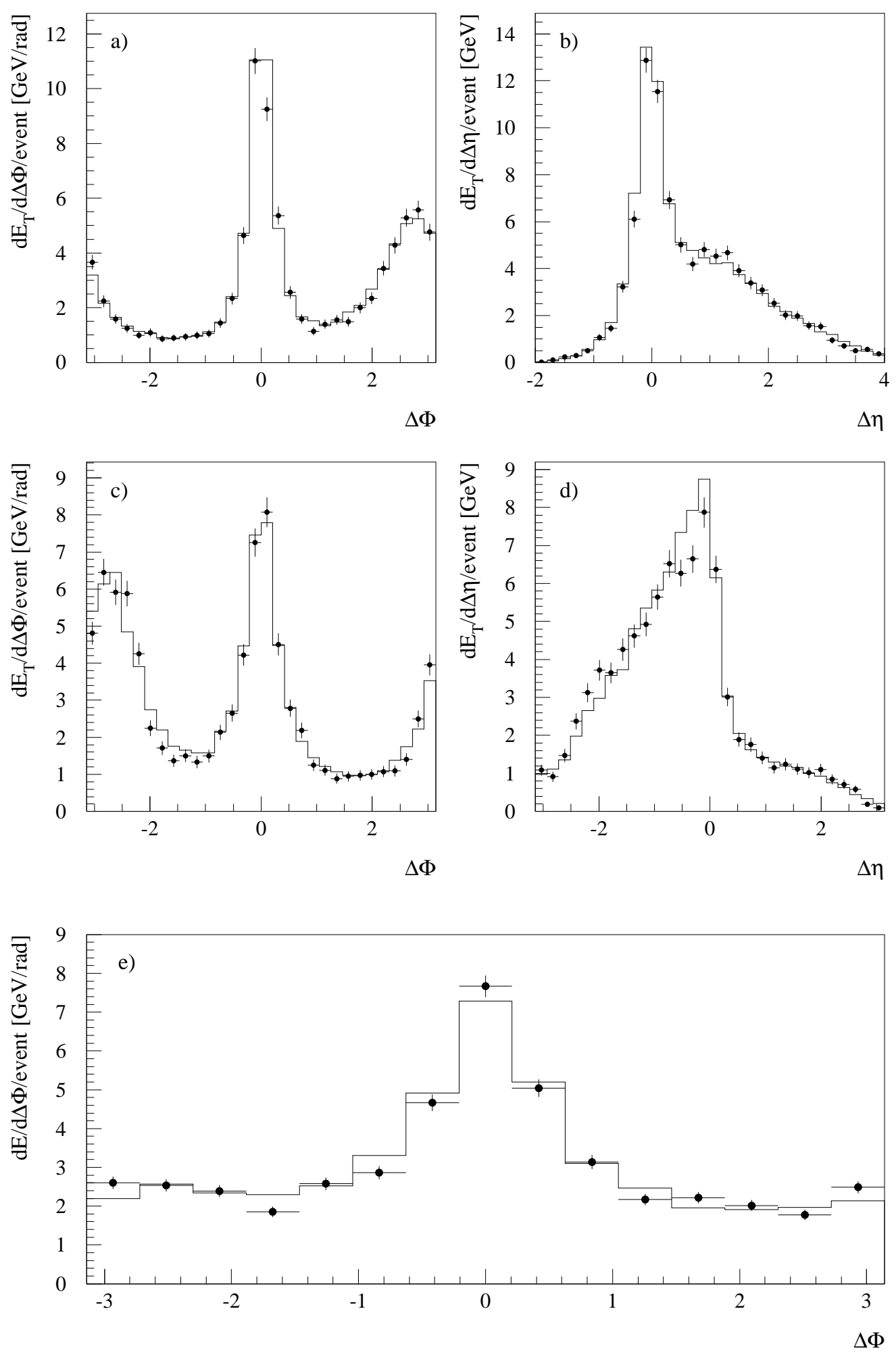

Figure 2: The transverse energy flow in the laboratory frame as a function of a) $\Delta \Phi$ and b) $\Delta \eta$ for the most backward going jet, and as a function of c) $\Delta \Phi$ and d) $\Delta \eta$ for the most forward going jet, using the respective jet axis as reference. The energy flow in the region $2<\eta<3$ is shown with the jet axis of the most forward going jet as reference in e). The sense of $\Phi$ is away from the scattered electron for the jet at the smallest $\eta$. The points represent the $(2+1)$ jet data sample and the errors are statistical whereas the histograms give the predictions of the MEPS model. 
covered by the matrix element. There is, however, some migration of events with invariant masses below $10 \mathrm{GeV}$. The contribution from the zeroth order process (QPM events) is about $60 \%$ at the generator level. In the final event sample a $2 \%$ contribution from these processes is observed, generated as $(1+1)$ jet events, but wrongly reconstructed as $(2+1)$ jet events. This indicates that the contribution from the phase space not covered by the matrix element is small.

Although the matrix element calculation used in LEPTO assumes massless quarks, the program contains a parametrisation which takes the heavy quark mass thresholds into account. In the kinematic region of this analysis, this effect reduces the LEPTO BGF cross section by $11 \%$ on the average.

A generated sample corresponding to about four times the statistics of the data was subject to full detector simulation, followed by event reconstruction.

A number of control plots have been made to compare the predictions of the Monte Carlo program with the experimental data. Fig. 2a-d show the transverse energy flow as a function of the azimuthal angle, $\Phi$, and pseudo-rapidity, $\eta$, for the two jets using the respective jet axis as reference. Particular attention was paid to the Monte Carlo description of the transverse energy flow in the forward region, $2<\eta<3$, which might contain a large contribution from initial state QCD radiation. Although we have previously observed [12] that the MEPS model is not able to reproduce the energy flow in this rapidity region for an inclusive DIS sample, it is demonstrated in Fig. 2le that the energy flow of the selected $(2+1)$ jet sample is well described by the MEPS model. It can be noted that not only the jet profile exhibits good agreement between data and the Monte Carlo sample but also the level of the underlying energy flow. Other control plots, such as the rapidity distributions of the two hard jets and their transverse energy spectra, as well as the $Q^{2}, W^{2}, y$ and $x$ distributions, give further evidence for the ability of the MEPS model to reproduce the data.

In order to estimate the model dependence of the BGF acceptance LEPTO MEPS was compared with other models available. It was found that the MEPS-, CDM 4.03- (colour dipole model) [13] and HERWIG 5.7 [14] models all give acceptances for BGF-events agreeing within $15 \%$ at the hadron level. The CDM model also gives a good description of the jet profiles but it is not able to give a satisfactory description of the cross section as a function of kinematic variables and jet variables as previously observed with less statistics in [15]. The discrepancies originate from non-BGF processes. In HERWIG there is no matching between the zeroth and first order processes, preventing an estimate of the migration of background.

\section{Reconstruction of $x_{g / p}$ and Unfolding of the Gluon Density}

The Monte Carlo sample was used to investigate the correlation between $x_{g / p}$ of the gluon calculated from the hard partons originating from the QCD matrix element and $x_{g / p}^{r e c}$

calculated from the jets measured in the detector. It is essential to use a jet reconstruction 

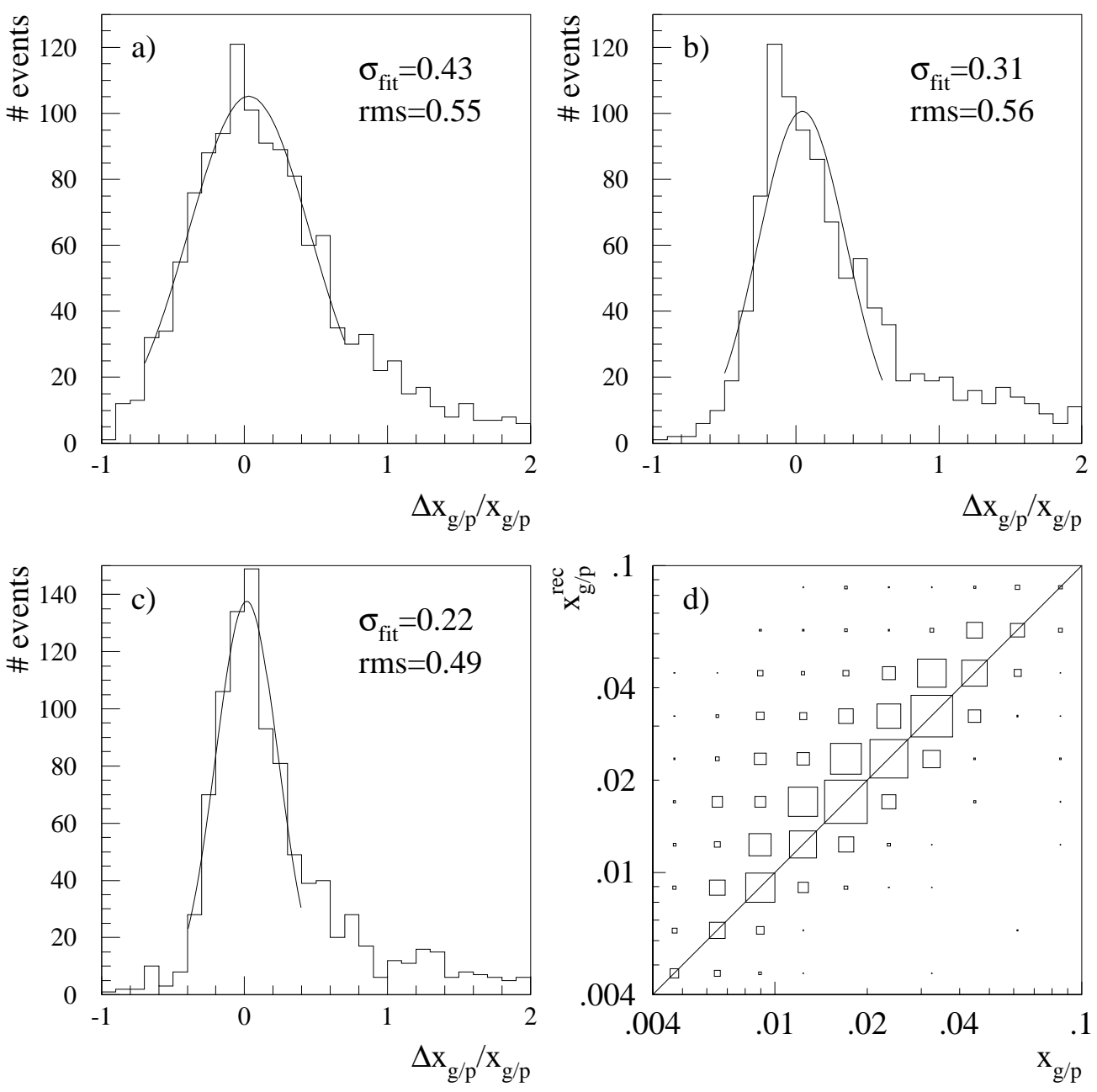

Figure 3: The relative error in the reconstruction of the fractional gluon momentum, $\Delta x_{g / p} / x_{g / p}$, where $\Delta x_{g / p}=x_{g / p}^{r e c}-x_{g / p}$, for BGF events generated by the MEPS model. In a) equation (国) has been used, in b) equation (3) and in c) the combination of the two. The correlation between the reconstructed and true $x_{g / p}$ for the combined method is shown in d).

algorithm which provides good separation between the spectator jet and the jets of the hard sub-system. The event sample populates the region of small $x$ and $Q^{2}$ values, the spectator jet will carry away most of the available energy. A misassignment of one of the relatively energetic particles from the spectator jet into the hard sub-system has a strong impact on the reconstructed $\hat{s}$ value and will distort the $x_{g / p}$ reconstruction. It is therefore important to optimize the resolution parameters of the jet algorithms to give the best possible performance in this respect.

The jet definition and detector effects lead to systematic shifts of the order of $30 \%$ in the determination of $x_{g / p}$ and $\hat{s}$ (see equation 1). The shifts have been determined separately for the two methods of extracting $x_{g / p}$ (equations (2) and (3)). Figs fa and $\mathrm{b}$ show the relative error in the $x_{g / p}$ reconstruction after correcting for the shift. 
Comparing the results of the two methods it can be seen that the fitted Gaussian distributions result in a superior resolution for the method based on equation (3) which, however, exhibits a more pronounced tail.

For properly reconstructed events both methods are expected to give consistent results, whereas a misassignment of particles by the jet algorithm might have different impacts on the $\hat{s}$ (and therefore $x_{g / p}$ ) reconstruction from the two methods. Also for $(1+1)$ jet events, where an additional jet arises from high $p_{T}$ initial parton emission, the methods do not give the same values of $\hat{s}$. Therefore an improved result is obtained by selecting only events for which the result of the two methods agree within the resolution. It has been demanded that the absolute value of the difference between the $\sqrt{\hat{s}}$ values extracted from equations (2) and (3) is $|\Delta \sqrt{\hat{s}}| \leq 10 \mathrm{GeV}$ and then simply taken the mean value of the two reconstructed values to give the combined $x_{g / p}$. Due to this cut about $20 \%$ of the events are removed.

As shown in Fig. [3c, the result of the combined method shows a considerably improved resolution compared with the methods based on equations (2) and (3) separately. In Fig. $3 \mathrm{~d}$ the correlation between the reconstructed and true $x_{g / p}$ is given for the combined method. From here on the combined method is used to extract $x_{g / p}$.

The observed $(2+1)$ jet cross section as a function of $x_{g / p}^{r e c}$ is interpreted as the sum of a gluon initiated (BGF) and a quark initiated (QCD-C plus QPM) part, according to

$$
\sigma_{o b s .}^{2+1}\left(x_{i / p}^{r e c}\right)=\int M\left(x_{g / p}, x_{g / p}^{r e c}\right) x_{g / p} g\left(x_{g / p}\right) \mathrm{d} x_{g / p}+\sigma_{M C}^{Q C D-C}\left(x_{q / p}^{r e c}\right)+\sigma_{M C}^{Q P M}\left(x_{q / p}^{r e c}\right)
$$

The quark initiated contributions represent the background obtained from the LEPTO Monte Carlo program. The integral represents the BGF part and expresses the convolution of the gluon density $x_{g / p} g\left(x_{g / p}\right)$ with the function $M$ which besides the measurement process describes effects due to the QCD matrix element, parton showering and hadronisation. The unfolding procedure with regularisation described in [16] is used to determine the gluon density. The $x_{g / p}$ range covered by the kinematic region is subdivided into five bins. The bin boundaries (see Tab. 1) were optimized in order to minimize the correlations between the bins.

\begin{tabular}{|c|c|c|c|c|}
\hline$x_{g / p}$-bin range & $x_{g / p} g\left(x_{g / p}\right)$ & $\left\langle Q^{2}>\left[\mathrm{GeV}^{2}\right]\right.$ & $\left.<p_{T}^{*}\right\rangle[\mathrm{GeV}]$ & $\begin{array}{c}\text { diffractive } \\
\text { contribution [\%] }\end{array}$ \\
\hline $0.0019-0.0061$ & $9.04 \pm 2.95$ & 23 & 5.0 & $16 \pm 9$ \\
$0.0061-0.012$ & $7.40 \pm 1.28$ & 29 & 5.4 & $10 \pm 5$ \\
$0.012-0.030$ & $4.03 \pm 1.06$ & 30 & 6.0 & $6 \pm 3$ \\
$0.030-0.052$ & $1.42 \pm 0.60$ & 35 & 6.6 & $9 \pm 4$ \\
$0.052-0.18$ & $1.18 \pm 0.80$ & 36 & 9.8 & $9 \pm 6$ \\
\hline
\end{tabular}

Table 1: Results and kinematic characteristics. Errors include statistical and systematic uncertainties, excluding a 11\% global normalization uncertainty. 


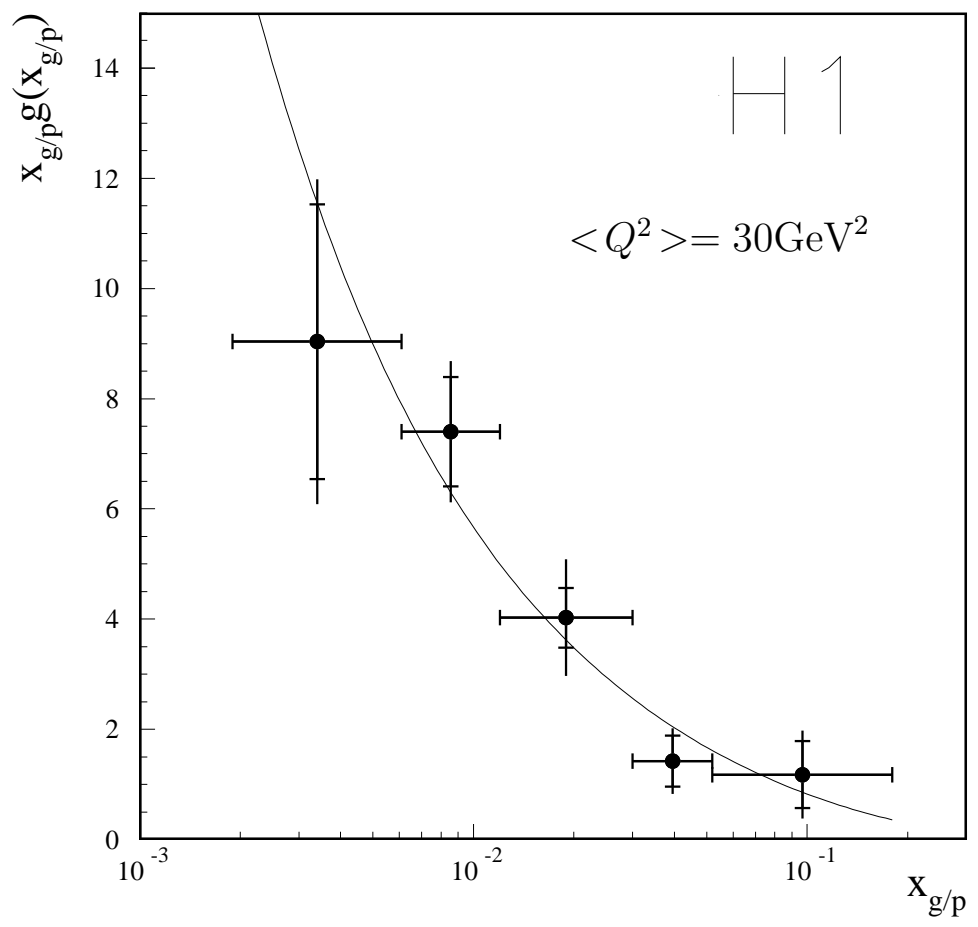

Figure 4: The measured gluon density at an average $Q^{2}$ of $30 \mathrm{GeV}^{2}$ as a function of the fractional gluon momentum. The error bars reflect the statistical errors and the total errors respectively. Not included is the global normalization uncertainty of 11\%. The solid curve shows a fit to the data points as explained in the text.

The results for the gluon density as a function of the fractional gluon momentum are presented in Fig. 4. The inner and outer error bars on the data points reflect the diagonal elements of the statistical and full covariance matrix respectively. Not shown is the $11 \%$ global normalization uncertainty. Additional information is given in Table 1. Results are quoted here at a scale of $30 \mathrm{GeV}^{2}$ corresponding to the average $Q^{2}$ value of the data sample. Another possible choice for the QCD-scale would be the average $p_{T}^{* 2}$ of the jets in the hadronic center-of-mass system, which for the present data sample is $40 \mathrm{GeV}^{2}$. Comparing the GRV gluon parametrisation [17] (see below) for $Q^{2}$ values of 20 and 40 $\mathrm{GeV}^{2}$ gives less than $10 \%$ variation in our range of $x_{g / p}$.

A considerable rise in the gluon content of the proton $x_{g / p} g\left(x_{g / p}\right)$ with decreasing fractional momentum of the gluon is observed. In Fig. 4 a fit of the data points to the parametrisation $x_{g / p} g\left(x_{g / p}\right)=A_{g} x_{g / p}^{B_{g}}\left(1-x_{g / p}\right)^{C_{g}}$ is shown taking the full covariance matrix into account (see Tab. 2). The parameter $C_{g}$ was kept fixed at the value of 5 . The values of the other parameters as obtained from the fit are $A_{g}=0.33 \pm 0.16$ and $B_{g}=-0.63 \pm 0.12$ with $\chi^{2} / n_{f}=0.99$. A value of $C_{g}=9$ lead to variations of $A_{g}$ and $B_{g}$ within the stated errors. 


\section{Discussion of Errors}

The unfolding yields a full covariance matrix of statistical errors which includes information on correlations between bins due to migration effects. In this analysis, the correlations are small (see Tab. 2), indicating adequate resolution for choosing five bins in $x_{g / p}$. For a quantitative estimate of systematic uncertainties we varied several parameters and repeated the full analysis including the unfolding step. For each variation, a covariance matrix was calculated. The total systematic covariance matrix is the sum of the matrices of the individual variations. The following effects were studied.

The absolute hadronic energy calibration (affecting the measurement of $p_{T}^{*}$ and $\hat{s}$ ) was varied by its present precision of $5 \%$. We also varied the electron energy calibration (affecting the measurement of the Bjørken variable $y$ and the total hadronic invariant mass $W$ ) by its measured precision of $\pm 1.7 \%$.

The jet transverse momentum cut in the cone algorithm used for jet finding was varied between 3 and $4 \mathrm{GeV}$. The jet angular cut $\Delta \eta$ nominally 2 was varied between 1.5 and 2.5 .

In section 5 it was shown that the MEPS model gives an excellent description of the data sample used in this analysis. However, comparing the distribution of the absolute difference of $\sqrt{\hat{s}}$ reconstructed according to equations (2) and (3) $(|\Delta \sqrt{\hat{s}}|$, see section 6$)$ an excess of data events is observed compared to the MEPS model at values of $|\Delta \sqrt{\hat{s}}|$ larger than $15 \mathrm{GeV}$. Below this value Monte Carlo and data show good agreement. The excess in data is due to events with at least one jet in the forward region, a region where the Monte Carlo description of data has been shown to be problematic [12]. However, even for the events rejected by the cut $|\Delta \sqrt{\hat{s}}|<10 \mathrm{GeV}$ the MEPS model gives a good description of the energy flows. For systematic studies, the $|\Delta \sqrt{\hat{s}}|$ cut is varied between 7 and $15 \mathrm{GeV}$.

QCD-Compton cross sections using two leading order (LO) quark density parametrisations (GRV [17] and CTEQ3L [18]) have been compared with the next to leading order (NLO) MRS H function used in the MC model. The discrepancies are largest at the low

$x_{q / p}$ but do not exceed $15 \%$ in the kinematic domain used. As a conservative estimate of the systematic uncertainty related to the subtraction of the QCD-Compton contribution a variation of $\pm 25 \%$ was used to determine the contribution to the systematic error.

$$
\left(\begin{array}{rrrrrr}
6.220 & & & & \\
0.087 & 0.978 & & & \\
-0.257 & -0.064 & 0.293 & & \\
0.033 & -0.065 & -0.017 & 0.214 & \\
0.032 & 0.013 & -0.049 & 0.003 & 0.366
\end{array}\right) \quad\left(\begin{array}{rrrrrr}
8.670 & & & & \\
0.858 & 1.632 & & & \\
0.976 & 0.584 & 1.117 & & \\
0.243 & 0.038 & 0.050 & 0.354 & \\
-0.407 & 0.108 & -0.162 & 0.044 & 0.642
\end{array}\right)
$$

Table 2: Covariance matrix of statistical errors (left) and total (sum of statistical and systematic) errors (right). Not included is a global normalization uncertainty of $11 \%$. 
Tab. 2 shows the total covariance matrix which is the sum of the statistical and systematic covariances. The relative systematic errors vary between $17 \%$ in the lowest bin of $x_{g / p}$ and $44 \%$ in the highest bin. The dominating contribution to the systematic errors in the lowest $x_{q / p}$ bin is $13 \%$ coming from the uncertainty in the hadronic energy calibration of the liquid argon calorimeter, while the dominating systematic error in the highest $x_{q / p}$ bin is $35 \%$ due to the variation of the $|\Delta \sqrt{\hat{s}}|$ cut.

In addition there is a global normalization uncertainty of $11 \%$ which arises from the following sources: a 4.5\% uncertainty in the luminosity measurement 19 directly propagates into the gluon density measurement, as does a $4 \%$ uncertainty in various detector efficiencies mentioned in section 4 . Finally, the one standard deviation uncertainty in the strong coupling constant $\alpha_{s}$ at $Q^{2}=M_{Z}^{2}$ [20] contributes a $9 \%$ uncertainty at $Q^{2}=30$ $\mathrm{GeV}^{2}$.

The variation of the parameters of the unfolding procedure within wide ranges had a negligible effect on the result.

The Monte Carlo model dependence of the BGF and QCD-Compton acceptances was estimated on the hadron level by comparing the numbers obtained from the CDM, HERWIG and MEPS models and was found to be below 15\% (see section 5).

The matrix element cut-off in the Monte Carlo generator on the hadron level was varied by $\pm 1 \mathrm{GeV}$ in order to study how it affects the event composition. No significant change was observed.

The radiative correction has been estimated for each $x_{g / p}$ bin by calculating the cross section with and without including QED radiation in the DJANGO 2.1 Monte Carlo program 21]. This led to variations consistent with the statistical accuracy of the generated Monte Carlo samples.

The full analysis was repeated using the JADE jet finding algorithm [22] in the laboratory frame with a fixed mass cut of $10 \mathrm{GeV}$. This is an algorithm based on a fundamentally different principle of reconstructing jets compared to the cone algorithm which results in different numbers of selected events and different migrations. In spite of this the finally obtained results on the gluon density agree within the statistical accuracy. The reason for using the cone algorithm to extract the final results was the better $x_{g / p}$ resolution and the greater suppression of tails.

As a further systematic check the analysis was repeated with a stricter cut in the invariant mass of the hard sub-system $\left(\hat{s}>200 \mathrm{GeV}^{2}\right)$, again yielding variations well within the statistical accuracy, although the statistical errors were significantly increased.

\section{Discussion of the Results}

So far only indirect constraints on the gluon density based on a $Q^{2}$ evolution of the quark density have been obtained for low values of $x$. A comparison between such indirect extraction and direct measurement of the gluon density constitutes an important test of 


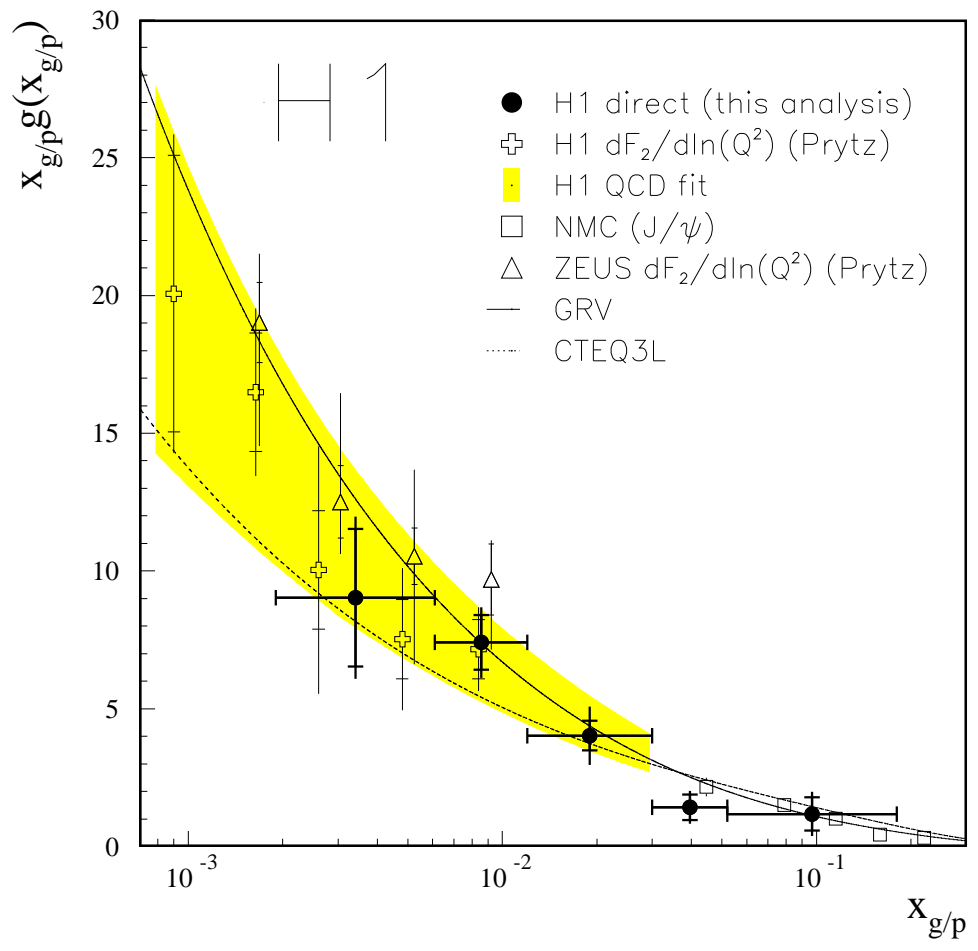

Figure 5: The measured gluon density at an average $Q^{2}$ of $30 \mathrm{GeV}^{2}$ as a function of the fractional gluon momentum compared with indirect determinations by $H 1$ [23] and ZEUS [24] at $Q^{2}=20 \mathrm{GeV}^{2}$ as well as with a determination from $\mathrm{J} / \Psi$ production by $\mathrm{NMC}$ [2X] evolved to $Q^{2}=30 \mathrm{GeV}^{2}$ (see text).

perturbative QCD. Fig. 5 shows a comparison of this measurement with recent indirect determinations by the H1 [23] and ZEUS [24] collaborations at a $Q^{2}$ of $20 \mathrm{GeV}^{2}$ which covers an even lower $x_{g / p}$ region. Our results are also consistent with a previous indirect measurement by NMC [25] in a region $x_{g / p}>10^{-2}$.

Direct measurements have been reported by UA2 [26] and NMC [27] at $x_{g / p}$ values above 0.04 . The NMC data based on inelastic $\mathrm{J} / \psi$ production in DIS is included in Fig 5. The parametrisation of the NMC data provided by this collaboration was used to perform a LO DGLAP evolution from $Q^{2}=1.5 \mathrm{GeV}^{2}$ to $Q^{2}=30 \mathrm{GeV}^{2}$.

Further the results are compared with two different parametrisations of the gluon density in LO. The GRV model assumes the gluons and sea quarks to be valence-like at $Q^{2}=0.23 \mathrm{GeV}^{2}$ and the growth of the gluon density with decreasing $x_{g / p}$ values is due to radiation of low $x$ partons generated according to the Altarelli-Parisi evolution equations. The CTEQ3L parametrisations are based on input distribution functions at $Q^{2}=4 \mathrm{GeV}^{2}$ assuming the sea and gluon distributions to have the same power dependence of the fractional momentum $\left(x^{B_{g}}\right)$. Both are consistent with our result.

The contribution of diffractive events, in which the exchanged boson interacts with a colourless object in the proton and therefore does not span a colour string between 
the hard sub-system and the proton, has been evaluated using data by selecting events with no activity in the forward detectors [28]. After correcting for the efficiency of the diffractive selection and for non diffractive events passing the selection cuts, a $(8 \pm 2) \%$ contribution is observed in the data sample consistent with a flat $x_{g / p}$ dependence (see also Table 1).

Next to leading order (NLO) corrections using the cone algorithm for jet production in deep-inelastic scattering do not exist. In photoproduction these corrections have been found to be of the order $25 \%$ for an average $\left\langle p_{T}^{*}\right\rangle$ (see Tab. 1) similar to this analysis [29]. Although in photoproduction besides the contribution from BGF and QCDC the so called resolved processes have been taken into account, NLO corrections of similar size are expected [30].

\section{Conclusions}

From a measurement of the cross section for $(2+1)$ jet events, a direct LO determination of the gluon density in the proton has been performed in a previously inaccessible domain of the gluon fractional momentum $x_{g / p}\left(1.9 \cdot 10^{-3}<x_{g / p}<0.18\right)$ at an average value of $Q^{2}=30 \mathrm{GeV}^{2}$. Our data are consistent with a steep rise in the gluon density as $x_{g / p}$

decreases. Recent indirect extractions of the gluon density based on the $Q^{2}$ evolution of the structure function $F_{2}$ by the ZEUS and $\mathrm{H} 1$ experiments are compatible with our data.

Acknowledgments: We are grateful to the HERA machine group whose outstanding efforts made this experiment possible. We appreciate the immense effort of the engineers and technicians who constructed and maintained the detector. We thank the funding agencies for financial support. We acknowledge the support of the DESY technical staff. We also wish to thank the DESY directorate for the hospitality extended to the non-DESY members of the collaboration. We have profited from many constructive discussions with members of the Uppsala and Lund theory groups.

\section{References}

[1] H1 Collaboration, T. Ahmed et al., Phys. Lett. B346 (1995) 415.

[2] New Muon Collaboration, P. Amaudruz et al., Phys. Lett. B295 (1992) 159;

[3] BCDMS Collaboration, A.C. Benvenuti et al., Phys. Lett. B223 (1989) 485.

[4] H1 Collaboration, I. Abt et al., The H1 Detector at HERA, DESY preprint 93-103.

[5] H1 Collaboration, I. Abt et al., Nucl. Phys. B493 (1995) 471.

[6] H1 Calorimeter Group, B. Andrieu et al., Nucl.Instrum.Meth. A336 (1993) 499. 
[7] CDF Collaboration, F. Abe et al., Phys. Rev. D45 (1992) 1448.

M. Seymour, Lund preprint LU TP 93-7, May 1993.

[8] G. Ingelman, LEPTO version 6.1, Uppsala internal report TSL/ISV-92-0065 and Proceedings 'Physics at HERA', Eds W. Buchmüller, G. Ingelman, DESY Hamburg 1992, vol. 3 and references therein.

[9] A.D. Martin, W.J. Stirling, R.G. Roberts, Phys. Rev. D50 (1994) 6743.

[10] D. Graudenz, PROJET 4.1, CERN-TH 7420/94.

[11] T. Brodkorb and E. Mirkes, DISJET program manual, MAD/PH/821, 1994.

[12] H1 Collaboration, I. Abt et al., Z. Phys. C 63 (1994) 377.

[13] L. Lönnblad, Computer Phys. Comm. 71 (1992) 15.

[14] G. Marchesini, B.R. Webber, G. Abbiendi, I.G. Knowles, M.H. Seymour, and L. Stanco, Computer Phys. Comm. 67 (1992) 465.

[15] H1 Collaboration, I. Abt et al. , Z. Phys. C61 (1994) 59.

[16] V. Blobel, DESY preprint 84-118, and Proceedings of the 1984 CERN School of Computing Aiguablava (Spain), CERN 1985.

[17] M. Glück, et al., Dynamical parton distributions of the proton and small x physics, DO-TH-94-24.

[18] H.L. Lai et al., Global QCD analysis and the CTEQ parton distributions, MSUHEP-41024.

[19] H1 Collaboration, T. Ahmed et al., Phys. Lett. B299 (1993) 374.

[20] Particle Data Group, L. Montanet et al., Phys. Rev. D50 (1994) 1173.

[21] G.A. Schuler and H. Spiesberger, 'Physics at HERA', Eds W. Buchmüller, G. Ingelman, DESY Hamburg 1992, vol. 3 and references therein.

[22] JADE Collaboration, W. Bartel et al., Z. Phys. C33 (1986) 23.

[23] H1 Collaboration, I. Abt et al., DESY preprint 95-081.

[24] ZEUS Collaboration, DESY preprint 94-192 (1994).

[25] New Muon Collaboration, M. Arneodo et al., Phys. Lett B309 (1993) 222.

[26] UA2 Collaboration, J. Alitti et al., Phys. Lett. B299 (1993) 174.

[27] New Muon Collaboration, D. Allasia et al., Phys. Lett. B258 (1991) 493.

[28] H1 Collaboration, I. Abt et al., Phys. Lett. B348 (1995) 681. 
[29] H1 Collaboration, T. Ahmed et al., DESY preprint 95-062, submitted to Nucl. Phys. B.

[30] L. E. Gordon \& J. K. Storrow, Phys. Lett. B291 (1992) 320. 